\title{
On Global properties of warped solutions in General Relativity with an electromagnetic field and a cosmological constant.
}

\author{
D. E. Afanasev, ${ }^{a}$ M. O. Katanaev ${ }^{* b c}$ \\ ${ }^{a}$ High school N1561, ul. Paustovskogo, 6, kor. 2, Moscow, 117464, Russia \\ ${ }^{b}$ Steklov Mathematical Institute, ul. Gubkina, 8, Moscow, 119991, Russia \\ ${ }^{c}$ N. I. Lobachevsky Institute of Mathematics and Mechanics, Kazan Federal University, \\ ul. Ktremlevskaya 35, Kazan, 420008, Russia \\ E-mail: daniel afanasevevahoo.com, katanaevdmi-ras.ru
}

\begin{abstract}
We consider general relativity with cosmological constant minimally coupled to the electromagnetic field and assume that the four-dimensional space-time manifold is a warped product of two surfaces with Lorentzian and Euclidean signature metrics. Einstein's equations imply that at least one of the surfaces must be of constant curvature. It means that the symmetry of the metric arises as the consequence of the equations of motion ("spontaneous symmetry emergence"). Totally, we have 37 topologically different global solutions with spatial symmetry. There is one solution among them describing changing topology of space in time which is discussed in detail.
\end{abstract}

Corfu Summer Institute 2019 "School and Workshops on Elementary Particle Physics and Gravity" (CORFU2019)

31 August - 25 September 2019

Corfu, Greece

\footnotetext{
* Speaker.
} 


\section{Introduction}

There are many well known exact solutions in general relativity (see, e.g. [四]). To give physical interpretation of any solution to Einstein's equation, we must know not only the metric satisfying equations of general relativity but the global structure of space-time. By this we mean a pair $(\mathbb{M}, g)$, where $\mathbb{M}$ is the four-dimensional space-time manifold and $g$ is the metric on $\mathbb{M}$ such, that manifold $\mathbb{M}$ is maximally extended along geodesics: any geodesic line on $\mathbb{M}$ either can be continued to infinite value of the canonical parameter in both directions, or it ends up at a singular point, where one of the geometric invariants becomes infinite. The famous example is the Kruskal-Szekeres extension [0, []] of the Schwarzschild solution. In this case, the space-time $\mathbb{M}$ is globally the topological product of a sphere (spherical symmetry) with the two-dimensional Lorentzian surface depicted by the well known Carter-Penrose diagram. The knowledge of this global structure of space-time allows one to introduce the notion of black and white holes.

The famous Reissner-Nordström solution [四, [] ], which is the spherically symmetric solution of Einstein's equations with electromagnetic field, is also known globally. There are three types of Carter-Penrose diagrams: the Reissner-Nordström black hole, extremal black hole and naked singularity. The type of the Carter-Penrose diagram depends on the relation between mass and charge parameters. The spherically symmetric exact solution of Einstein's equations with electromagnetic field and cosmological constant is known locally but not analyzed in full detail globally. Recently, we give complete classification of global spherically symmetric solutions of Einstein's equations with electromagnetic field and cosmological constant, which depends on relations between three parameters: mass, charge, and cosmological constant [6]. We showed that there are 16 different Carter-Penrose diagrams in the spherically symmetric case.

In fact, more general classification is given. We do not assume that solutions have any symmetry from the very beginning. Instead, we require the space-time to be the warped product of two surfaces: $\mathbb{M}=\mathbb{U} \times \mathbb{V}$, where $\mathbb{U}$ and $\mathbb{V}$ are two two-dimensional surfaces with Lorentzian and Euclidean signature metrics, respectively. As the consequence of the equations of motion, at least one of the surfaces must be of constant curvature. In this paper, we consider the cases when (i) both surfaces $\mathbb{U}$ and $\mathbb{V}$ are of constant curvature and when (ii) only surface $\mathbb{V}$ is of constant curvature. In the latter case, there are three possibilities: $\mathbb{V}$ is the sphere $\mathbb{S}^{2}$ (the spherical $\mathbb{S O}(3)$ symmetry), the Euclidean plane (the Poincare $\mathbb{I S O}(2)$ symmetry), and the two-sheeted hyperboloid $\mathbb{H}^{2}$ (the Lorentzian $\mathbb{S O}(1,2)$ symmetry). We see that the symmetry of solutions is not assumed from the beginning but arise as the consequence of the equations of motions. This effect is called "spontaneous symmetry emergence". All global solutions are classified by drawing their Carter-Penrose diagrams for surface $\mathbb{U}$ depending on relations between mass, charge, and cosmological constant [四]. Totally, there are 4 different Carter-Penrose diagrams in case (i) and 33 globally different solutions in case (ii).

Moreover, we prove that there is the additional fourth Killing vector field in case (ii). This is a generalization of Birkhoff's theorem stating that any spherically symmetric solution of vacuum Einstein's equations must be static. The existence of extra Killing vector field is proved for $\mathbb{S O}(3)$, $\mathbb{I S O}(2)$, and $\mathbb{S O}(1,2)$ symmetry groups.

Global structure of space-times in general relativity and 2d-gravity was analyzed, e.g. in [711]. In particular, global planar and Lobachevsky plane solutions in general relativity were de- 
scribed in [12], [3], [4]]. Though many solutions in the paper are known globally, some of them seem to be new. For example, the Carter-Penrose diagram with triple horizon and geodesically complete central point is new to the best of our knowledge. It describes changing topology of space in time and discussed in some detail.

The paper [四] follows the classification of global warped product solutions of general relativity with cosmological constant (without electromagnetic field) given in [15]]. The Carter-Penrose diagrams are constructed using the conformal block method described in [ए6].

As in [피], we assume that space-time $\mathbb{M}$ is the warped product of two surfaces: $\mathbb{M}=\mathbb{U} \times \mathbb{V}$, where $\mathbb{U}$ and $\mathbb{V}$ are surfaces with Lorentzian and Euclidean signature metrics, respectively. Local coordinates on $\mathbb{M}$ are denoted by $\hat{x}^{i}, i=0,1,2,3$, and coordinates on the surfaces by Greek letters from the beginning and middle of the alphabet:

$$
\left(x^{\alpha}\right) \in \mathbb{U}, \quad \alpha=0,1, \quad\left(y^{\mu}\right) \in \mathbb{V}, \quad \mu=2,3 .
$$

That is $\left(\hat{x}^{i}\right):=\left(x^{\alpha}, y^{\mu}\right)$. Geometrical notions on four-dimensional space-time are marked by the hat to distinguish them from notions on surfaces $\mathbb{U}$ and $\mathbb{V}$, which appear more often.

We do not assume any symmetry of solutions from the very beginning.

Four-dimensional metric of the warped product of two surfaces has block diagonal form by definition:

$$
\widehat{g}_{i j}=\left(\begin{array}{cc}
k(y) g_{\alpha \beta}(x) & 0 \\
0 & m(x) h_{\mu v}(y)
\end{array}\right),
$$

where $g_{\alpha \beta}(x)$ and $h_{\mu v}(y)$ are some metrics on surfaces $\mathbb{U}$ and $\mathbb{V}$, respectively, $k(y) \neq 0$ and $m(x) \neq 0$ are scalar (dilaton) fields on $\mathbb{V}$ and $\mathbb{U}$. Without loss of generality, signatures of two-dimensional metrics $g_{\alpha \beta}$ and $h_{\mu \nu}$ are assumed to be $(+-)$ and $(++)$, respectively. In a rigorous sense, metric (ㄸ. $)$ is a doubly warped product. It reduces to a warped product in the usual sense for $k=$ const or $m=$ const.

The Ricci tensor components for metric (미) are

$$
\begin{aligned}
& \widehat{R}_{\alpha \beta}=R_{\alpha \beta}+\frac{\nabla_{\alpha} \nabla_{\beta} m}{m}-\frac{\nabla_{\alpha} m \nabla_{\beta} m}{2 m^{2}}+\frac{g_{\alpha \beta} \nabla^{2} k}{2 m} \\
& \widehat{R}_{\alpha \mu}=\widehat{R}_{\mu \alpha}=-\frac{\nabla_{\alpha} m \nabla_{\mu} k}{2 m k} \\
& \widehat{R}_{\mu v}=R_{\mu v}+\frac{\nabla_{\mu} \nabla_{v} k}{k}-\frac{\nabla_{\mu} k \nabla_{v} k}{2 k^{2}}+\frac{h_{\mu v} \nabla^{2} m}{2 k},
\end{aligned}
$$

where, for brevity, we introduce notation

$$
\nabla^{2} m:=g^{\alpha \beta} \nabla_{\alpha} \nabla_{\beta} m, \quad \nabla^{2} k:=h^{\mu v} \nabla_{\mu} \nabla_{v} k
$$

Here and in what follows symbol $\nabla$ denotes covariant derivative with the corresponding Christoffel's symbols. The four-dimensional scalar curvature is

$$
\widehat{R}=\frac{1}{k} R^{(g)}+2 \frac{\nabla^{2} m}{k m}-\frac{(\nabla m)^{2}}{2 k m^{2}}+\frac{1}{m} R^{(h)}+2 \frac{\nabla^{2} k}{k m}-\frac{(\nabla k)^{2}}{2 k^{2} m},
$$

where

$$
(\nabla m)^{2}:=g^{\alpha \beta} \partial_{\alpha} m \partial_{\beta} m, \quad(\nabla k)^{2}:=h^{\mu v} \partial_{\mu} k \partial_{v} k .
$$

Scalar curvatures of surfaces $\mathbb{U}$ and $\mathbb{V}$ are denoted by $R^{(g)}$ and $R^{(h)}$, respectively. 


\section{Solution for electromagnetic field}

We assume that electromagnetic field is minimally coupled to gravity. Then the action takes the form

$$
S=\int d^{4} x \sqrt{|\widehat{g}|}\left(\widehat{R}-2 \Lambda-\frac{1}{4} \widehat{F}^{2}\right)
$$

where $\widehat{R}$ is the scalar curvature for metric $\widehat{g}_{i j}, \widehat{g}:=\operatorname{det} \widehat{g}_{i j}, \Lambda$ is a cosmological constant, and $\widehat{F}^{2}$ is the square of electromagnetic field strength:

$$
\widehat{F}^{2}:=\widehat{F}_{i j} \widehat{F}^{i j}, \quad \widehat{F}_{i j}:=\partial_{i} \widehat{A}_{j}-\partial_{j} \widehat{A}_{i} .
$$

Here, $\widehat{A}_{i}$ are components of electromagnetic field potential. For brevity, gravitational and electromagnetic coupling constants are set to unity.

Variation of action (‥ (ل) with respect to metric yields four-dimensional Einstein's equations:

$$
\widehat{R}_{i j}-\frac{1}{2} \widehat{g}_{i j} \widehat{R}+\widehat{g}_{i j} \Lambda=-\frac{1}{2} \widehat{T}_{\mathrm{EM} i j}
$$

where

$$
\widehat{T}_{\mathrm{EM} i j}:=-\widehat{F}_{i k} \widehat{F}_{j}^{k}+\frac{1}{4} \widehat{g}_{i j} \widehat{F}^{2}
$$

is the electromagnetic field energy-momentum tensor. Variation of the action with respect to electromagnetic field yields Maxwell's equations:

$$
\partial_{j}\left(\sqrt{|\widehat{g}|} \widehat{F}^{j i}\right)=0
$$

where

$$
\widehat{g}=k^{2} m^{2} g h, \quad g:=\operatorname{det} g_{\alpha \beta}, \quad h:=\operatorname{det} h_{\mu v} .
$$

To simplify the problem, we assume that the four-dimensional electromagnetic potential consists of two parts:

$$
\widehat{A}_{i}=\left(A_{\alpha}(x), A_{\mu}(y)\right),
$$

where $A_{\alpha}(x)$ and $A_{\mu}(y)$ are two-dimensional electromagnetic potentials on surfaces $\mathbb{U}$ and $\mathbb{V}$, respectively. Then the electromagnetic field strength becomes block diagonal:

$$
\widehat{F}_{i j}=\left(\begin{array}{cc}
F_{\alpha \beta} & 0 \\
0 & F_{\mu v}
\end{array}\right),
$$

where

$$
F_{\alpha \beta}(x):=\partial_{\alpha} A_{\beta}-\partial_{\beta} A_{\alpha}, \quad F_{\mu v}(y):=\partial_{\mu} A_{v}-\partial_{v} A_{\mu}
$$

are strength components for two-dimensional electromagnetic potentials.

In what follows, the raising of Greek indices from the beginning and middle of the Greek alphabet is performed by using the inverse metrics $g^{\alpha \beta}$ and $h^{\mu v}$. Therefore

$$
\widehat{F}^{\alpha \beta}=\frac{1}{k^{2}} F^{\alpha \beta}, \quad \widehat{F}^{\mu v}=\frac{1}{m^{2}} F^{\mu v},
$$


where $k(y)$ and $m(x)$ are dilaton fields entering four-dimensional metric $(\mathbb{L} \mathbb{C})$. The square of fourdimensional electromagnetic field strength is

$$
\widehat{F}^{2}=\frac{1}{k^{2}} F_{\alpha \beta} F^{\alpha \beta}+\frac{1}{m^{2}} F_{\mu \nu} F^{\mu \nu} .
$$

In the case under consideration, Maxwell's Eqs. (2.4) for $i=\alpha$ lead to equality

$$
\frac{1}{|k|} \sqrt{|h|} \partial_{\beta}\left(|m| \sqrt{|g|} F^{\beta \alpha}\right)=0 .
$$

A general solution to these equations has the form

$$
|m| \sqrt{|g|} F^{\alpha \beta}=2 \hat{\varepsilon}^{\alpha \beta} Q, \quad Q=\text { const },
$$

where $\hat{\varepsilon}^{\alpha \beta}$ is the totally antisymmetric second rank tensor density. The factor 2 is introduced in the right hand side of general solution for simplification of subsequent formulae. This solution is rewritten as

$$
F^{\alpha \beta}=\frac{2 Q}{|m|} \varepsilon^{\alpha \beta}
$$

where $\varepsilon^{\alpha \beta}:=\hat{\varepsilon}^{\alpha \beta} / \sqrt{|g|}$ is now the totally antisymmetric second rank tensor.

If $i=\mu$, then Maxwell's Eqs. (2.4) yield the equality

$$
\frac{1}{|m|} \sqrt{|g|} \partial_{\mu}\left(|k| \sqrt{h} F^{\mu v}\right)=0 .
$$

Its general solution is

$$
F^{\mu v}=\frac{2 P}{|k|} \varepsilon^{\mu v}, \quad P=\text { const. }
$$

Now the four-dimensional electromagnetic energy-momentum tensor ([2.3]) is easily calculated. It is block diagonal:

$$
\widehat{T}_{i j}=\left(\begin{array}{cc}
\widehat{T}_{\alpha \beta} & 0 \\
0 & \widehat{T}_{\mu v}
\end{array}\right),
$$

where

$$
\widehat{T}_{\alpha \beta}=\frac{2 g_{\alpha \beta}}{k m^{2}}\left(Q^{2}+P^{2}\right), \quad \widehat{T}_{\mu v}=-\frac{2 h_{\mu v}}{k^{2} m}\left(Q^{2}+P^{2}\right) .
$$

Now we have to solve Einstein's Eqs. (2.2) with right hand side (2.9). Since energy-momentum tensor depends only on the sum $Q^{2}+P^{2}$, we set $P=0$ to simplify formulae. In the final answer, this constant is easily reconstructed by substitution $Q^{2} \mapsto Q^{2}+P^{2}$.

In what follows, we consider only the case $Q \neq 0$, because the case $Q=0$ was considered in [ए5]] in full detail.

\section{Einstein's equations}

The right hand side of Einstein's Eqs. (2.2) is defined by general solution of Maxwell's equations, which leads to electromagnetic energy-momentum tensor (2. $)$. The trace of Einstein's equations can be easily solved with respect to the scalar curvature: $\widehat{R}=4 \Lambda$. After elimination of the scalar curvature, Einstein's equations are simplified:

$$
\widehat{R}_{i j}-\widehat{g}_{i j} \Lambda=-\frac{1}{2} \widehat{T}_{\mathrm{EM} i j}
$$


For indices values $(i j)=(\alpha, \beta),(\mu v)$, and $(\alpha, \mu)$, these equations yield the following system of equations:

$$
\begin{aligned}
R_{\alpha \beta}+\frac{\nabla_{\alpha} \nabla_{\beta} m}{m}-\frac{\nabla_{\alpha} m \nabla_{\beta} m}{2 m^{2}}+g_{\alpha \beta}\left(\frac{\nabla^{2} k}{2 m}-k \Lambda+\frac{Q^{2}}{m^{2} k}\right) & =0, \\
R_{\mu v}+\frac{\nabla_{\mu} \nabla_{v} k}{k}-\frac{\nabla_{\mu} k \nabla_{v} k}{2 k^{2}}+h_{\mu v}\left(\frac{\nabla^{2} m}{2 k}-m \Lambda-\frac{Q^{2}}{k^{2} m}\right) & =0, \\
-\frac{\nabla_{\alpha} m \nabla_{\mu} k}{2 m k} & =0,
\end{aligned}
$$

where $R_{\alpha \beta}$ and $R_{\mu v}$ are Ricci tensors for two-dimensional metrics $g_{\alpha \beta}$ and $h_{\mu v}$, respectively, $\nabla_{\alpha}$ and $\nabla_{\mu}$ are two-dimensional covariant derivatives with Christoffel's symbols on surfaces $\mathbb{U}$ and $\mathbb{V}, \nabla^{2}:=g^{\alpha \beta} \nabla_{\alpha} \nabla_{\beta}$ or $\nabla^{2}:=h^{\mu \nu} \nabla_{\mu} \nabla_{v}$, which is clear from the context. Sure, the equalities $\nabla_{\alpha} m=\partial_{\alpha} m$ and $\nabla_{\mu} k=\partial_{\mu} k$ hold. But we keep the symbol of covariant derivative for uniformity.

For subsequent analysis of Einstein's equations, we extract the traces and traceless parts from Eqs. (B.2) and (B.3). Then the full system of Einstein's equations takes the form

$$
\begin{aligned}
\nabla_{\alpha} \nabla_{\beta} m-\frac{\nabla_{\alpha} m \nabla_{\beta} m}{2 m}-\frac{1}{2}\left(\nabla^{2} m-\frac{(\nabla m)^{2}}{2 m}\right) & =0, \\
\nabla_{\mu} \nabla_{v} k-\frac{\nabla_{\mu} k \nabla_{v} k}{2 k}-\frac{1}{2}\left(\nabla^{2} k-\frac{(\nabla k)^{2}}{2 k}\right) & =0, \\
R^{(g)}+\frac{\nabla^{2} m}{m}-\frac{(\nabla m)^{2}}{2 m^{2}}+\frac{\nabla^{2} k}{m}-2 k \Lambda+\frac{2 Q^{2}}{m^{2} k} & =0, \\
R^{(h)}+\frac{\nabla^{2} k}{k}-\frac{(\nabla k)^{2}}{2 k^{2}}+\frac{\nabla^{2} m}{k}-2 m \Lambda-\frac{2 Q^{2}}{k^{2} m} & =0, \\
\nabla_{\alpha} m \nabla_{\beta} k & =0,
\end{aligned}
$$

where $(\nabla m)^{2}:=g^{\alpha \beta} \nabla_{\alpha} m \nabla_{\beta} m,(\nabla k)^{2}:=g^{\mu \nu} \nabla_{\mu} k \nabla_{v} k, R^{(g)}$ and $R^{(h)}$ are scalar curvatures of twodimensional surfaces $\mathbb{U}$ and $\mathbb{V}$ for metrics $g$ and $h$, respectively. In the above formulae, we used equalities $R_{\alpha \beta}=\frac{1}{2} g_{\alpha \beta} R^{(g)}$ and $R_{\mu \nu}=\frac{1}{2} h_{\mu \nu} R^{(h)}$ valid in two dimensions.

The last Eq. (B.9), which corresponds to mixed values of indices $(i j)=(\alpha \mu)$ in Einstein's equations results in strong restrictions on solutions. Namely, as in the case without electromagnetic field, there are only three cases:

$$
\begin{array}{lrr}
\text { A : } & k=\text { const } \neq 0, \quad m=\text { const } \neq 0, \\
\text { B : } & k=\text { const } \neq 0, & \nabla_{\alpha} m \neq 0, \\
\text { C }: & \nabla_{\mu} k \neq 0, & m=\text { const } \neq 0 .
\end{array}
$$

We shall see in what follows, that this leads to "spontaneous symmetry emergence".

Now we consider the first two cases in detail.

\section{Product of constant curvature surfaces}

The most symmetric solutions of Einstein's equations with electromagnetic field in the form of the product of two constant curvature surfaces arise in case $A(B . T)$ ), when both dilaton fields are 
constant. If $k$ and $m$ are constant, then Eqs. (B.5) and (B.6) are identically satisfied, and Eqs. (3.7) and (B.8) take the form

$$
R^{(g)}=2 k \Lambda-\frac{2 Q^{2}}{m^{2} k}=-2 K^{(g)}, \quad R^{(h)}=2 m \Lambda+\frac{2 Q^{2}}{k^{2} m}=-2 K^{(h)},
$$

where

$$
K^{(g)}:=-k\left(\Lambda-\frac{Q^{2}}{k^{2} m^{2}}\right), \quad K^{(h)}:=-m\left(\Lambda+\frac{Q^{2}}{k^{2} m^{2}}\right)
$$

are Gaussian curvatures of surfaces $\mathbb{U}$ and $\mathbb{V}$, respectively. It means that both surfaces are of constant curvature in case $A$. The metric on each surface is invariant under three-dimensional transformation group.

In stereographic coordinates on both surfaces, the metric of four-dimensional space-time takes the form

$$
\begin{aligned}
d s^{2} & =k g_{\alpha \beta} d x^{\alpha} d x^{\beta}+m h_{\mu v} d y^{\mu} d y^{v}= \\
& =k \frac{d t^{2}-d x^{2}}{\left[1+\frac{K^{(g)}}{4}\left(t^{2}-x^{2}\right)\right]^{2}}+m \frac{d y^{2}+d z^{2}}{\left[1+\frac{K^{(h)}}{4}\left(y^{2}+z^{2}\right)\right]^{2}},
\end{aligned}
$$

where $\left(x^{\alpha}\right):=(t, x)$ and $\left(y^{\mu}\right):=(y, z)$.

We can put $k= \pm 1$ and $m= \pm 1$ by rescaling coordinates. One has also to redefine the constant of integration $Q^{2} /\left(k^{2} m^{2}\right) \mapsto Q^{2}$. We choose $k=1$ and $m=-1$ for the metric signature to be $(+---)$. Then the Gaussian curvatures are

$$
K^{(g)}=Q^{2}-\Lambda, \quad K^{(h)}=Q^{2}+\Lambda .
$$

There are four qualitatively different cases for topologically inequivalent global solutions depending on relations between cosmological constant and charge:

$$
\begin{array}{rlll}
\Lambda<-Q^{2}: & K^{(g)}>0, & K^{(h)}<0, & \mathbb{M}=\mathbb{L}^{2} \times \mathbb{H}^{2}, \\
\Lambda=-Q^{2}: & K^{(g)}>0, & K^{(h)}=0, & \mathbb{M}=\mathbb{L}^{2} \times \mathbb{R}^{2}, \\
-Q^{2}<\Lambda<Q^{2}: & K^{(g)}>0, & K^{(h)}>0, & \mathbb{M}=\mathbb{L}^{2} \times \mathbb{S}^{2}, \\
\Lambda=Q^{2}: & K^{(g)}=0, & K^{(h)}>0, & \mathbb{M}=\mathbb{R}^{1,1} \times \mathbb{S}^{2}, \\
\Lambda>Q^{2}: & K^{(g)}<0, & K^{(h)}>0, & \mathbb{M}=\mathbb{L}^{2} \times \mathbb{S}^{2},
\end{array}
$$

where $\mathbb{L}^{2}$ is the one sheet hyperboloid (more precisely, its universal covering) embedded in threedimensional Minkowskian space $\mathbb{R}^{1,2}, \mathbb{H}^{2}$ is the Lobachevsky plane (the upper sheet of the twosheeted hyperboloid embedded in $\mathbb{R}^{1,2}$ ), and $\mathbb{S}^{2}$ is the two-dimensional sphere. From topological point of view the third and fifth cases in Eq. (4.4) coincide. Therefore there are only four topologically inequivalent global solutions of Einstein's equations in the form of direct product of two constant curvature surfaces. Note that for $Q=0$, there are only three topologically inequivalent solutions [피].

All solutions have exactly six Killing vector fields and belong to type $D$ in Petrov's classification.

The cases of other signatures of four-dimensional metric for $k= \pm 1$ and $m= \pm 1$ are analysed similarly. Qualitative properties of global solutions are the same.

We see that symmetry properties in this case are not imposed from the very beginning but arise as the result of solution of equations of motion. This effect is called "spontaneous symmetry emergence". 


\section{Solutions with spatial symmetry}

The dilaton field $k$ is constant in second case B (B.J)). Without loss of generality, we put $k=1$. Then Einstein's equations (B.5) - (B.9) take the form

$$
\begin{aligned}
\nabla_{\alpha} \nabla_{\beta} m-\frac{\nabla_{\alpha} m \nabla_{\beta} m}{2 m}-\frac{1}{2} g_{\alpha \beta}\left[\nabla^{2} m-\frac{(\nabla m)^{2}}{2 m}\right] & =0, \\
R^{(h)}+\nabla^{2} m-2 m \Lambda-\frac{2 Q^{2}}{m} & =0, \\
R^{(g)}+\frac{\nabla^{2} m}{m}-\frac{(\nabla m)^{2}}{2 m^{2}}-2 \Lambda+\frac{2 Q^{2}}{m^{2}} & =0 .
\end{aligned}
$$

Consider Eq. (5.2). The scalar curvature $R^{(h)}$ depends on coordinates $y$ on surface $\mathbb{V}$, whereas all other terms depend on coordinates $x$ on surface $\mathbb{U}$. For this equation to be fulfilled, it is necessary that equation $R^{(h)}=$ const holds. It means that surface $\mathbb{V}$ must be of constant curvature as the consequence of Einstein equations. Therefore the four-dimensional metric of space-time has at least three independent Killing vector fields. So, there is spontaneous symmetry emergence.

Let us put $R^{(h)}:=-2 K^{(h)}=$ const. Then Eq. (5.2) is

$$
\nabla^{2} m-2 m \Lambda-2 K^{(h)}-\frac{2 Q^{2}}{m}=0 .
$$

Excluding the case $A$ considered in the previous section, we proceed further assuming $\nabla_{\alpha} m \neq$ 0 on the whole $\mathbb{U}$.

Proposition 5.1. Equation (5.4) is the first integral of Eqs. (5. (15) and (5.3)).

The proof is given in [团]. As the consequence, it is sufficient to solve Eqs. (5.]) and (5.4), Eq. (5.3) being satisfied automatically.

To solve Eqs. (للـ ) and (5. explicitly, we fix the conformal gauge for metric $g_{\alpha \beta}$ on Lorentzian surface $\mathbb{U}$ :

$$
g_{\alpha \beta} d x^{\alpha} d x^{\beta}=\Phi d \xi d \eta
$$

where $\Phi(\xi, \eta) \neq 0$ is the conformal factor depending on light cone coordinates $\xi:=\tau+\sigma, \eta:=$ $\tau-\sigma$ on $\mathbb{U}$. The respective four dimensional metric is

$$
d s^{2}=\Phi d \xi d \eta+m d \Omega^{2},
$$

where $d \Omega^{2}$ is the metric on the Riemannian surface of constant curvature $\mathbb{V}=\mathbb{S}^{2}, \mathbb{R}^{2}$, or $\mathbb{H}^{2}$. The sign of the conformal factor $\Phi$ is not fixed for the present.

For $\Phi>0$ and $m<0$ the signature of metric (5.6) is $(+---)$. If we change the sign of $m$, the signature of the metric becomes $(+-++)$. The same transformation of the signature can be achieved by changing the overall sign of the metric $\widehat{g}_{i j} \mapsto-\widehat{g}_{i j}$, and interchanging the first two coordinates, $\tau \leftrightarrow \sigma$. Einstein's equations with cosmological constant and electromagnetic field (B. $)$ are not invariant with respect to these transformations with simultaneous changing the sign of the cosmological constant, because the right hand side changes its sign. Therefore, for $\Phi>0$, we have to consider two cases:

$$
m<0 \quad \Leftrightarrow \quad \operatorname{sign} \widehat{g}_{i j}=(+---) \quad \text { and } \quad m>0 \quad \Leftrightarrow \quad \operatorname{sign} \widehat{g}_{i j}=(-+++) .
$$


This is the difference for Einstein's equations without electromagnetic field considered in [디]. If the change of signature $(+---) \mapsto(-+++)$ is followed by the change of signs in front of $\widehat{R}, \Lambda$, and $\widehat{F}^{2}$ in action $(\mathbb{R} . \mathbb{C})$, then both choices become equivalent. In our presentation, it is more natural to fix the sign of $\widehat{F}^{2}$ in action (ZD) and consider different signatures, because signs of dilaton fields are not fixed.

For $\Phi>0$ and $m<0$, the metric signature is (+- - ), and we introduce convenient parameterization

$$
m:=-q^{2}, \quad q(\xi, \eta)>0
$$

Afterwards, we obtain the full system of equations:

$$
\begin{array}{r}
-\partial_{\xi \xi}^{2} q+\frac{\partial_{\xi} \Phi \partial_{\xi} q}{\Phi}=0 \\
-\partial_{\eta \eta}^{2} q+\frac{\partial_{\eta} \Phi \partial_{\eta} q}{\Phi}=0 \\
-2 \frac{\partial_{\xi \eta}^{2} q^{2}}{\Phi}-K^{(h)}+\Lambda q^{2}+\frac{Q^{2}}{q^{2}}=0
\end{array}
$$

The first two equations which do not depend on the electromagnetic field imply the following assertion.

Proposition 5.2. If $\partial_{\xi} q \partial_{\eta} q>0$, then the function $q(\tau)$ depends only on timelike coordinate $\tau:=$ $\frac{1}{2}(\xi+\eta)$. If $\partial_{\xi} q \partial_{\eta} q<0$, then the function $q(\sigma)$ depends only on spacelike coordinate $\sigma:=$ $\frac{1}{2}(\xi-\eta)$. And the following equality holds

$$
|\Phi|=\left|q^{\prime}\right|
$$

where prime denotes differentiation on the argument (either $\tau$, or $\sigma$ ).

This proposition provides a general solution to equations (5.8) and (5.9) up to conformal transformations. This statement is proved in [45, [1]].

Thus, we can always choose coordinates in such a way that $q$ and $\Phi$ depend simultaneously on timelike or spacelike coordinate

$$
\zeta:=\frac{1}{2}(\xi \pm \eta)=: \begin{cases}\tau, & \partial_{\xi} q \partial_{\eta} q>0 \\ \sigma, & \partial_{\xi} q \partial_{\eta} q<0 .\end{cases}
$$

It means that two-dimensional metric (5.5) and consequently four-dimensional metric (5.6) have the Killing vector $\partial_{\sigma}$ or $\partial_{\tau}$, as the consequence of equations (5.8) and (5.9). We call these solutions homogeneous and static, respectively, though it is related to the fixed coordinate system. The existence of additional Killing vector is the generalization of Birkhoff's theorem [ए8] stating that arbitrary spherically symmetric solution of vacuum Einstein's equations must be static. (This statement was previously published in [ए9].) The generalization includes the addition of electromagnetic field, and, in addition, the existence of extra Killing vector is proved not only for spherically symmetric solution $\left(K^{(h)}=1\right)$, but also for solutions invariant with respect to $\operatorname{ISO}(2)$ $\left(K^{(h)}=0\right)$ and $\mathbb{S O}(1,2)\left(K^{(h)}=-1\right)$ transformation groups. 
We are left to solve equation (1) $)$. In static, $q=q(\sigma)$, and homogeneous, $q=q(\tau)$, cases, equation (5.T0) takes the form

$$
\begin{array}{ll}
\left(q^{2}\right)^{\prime \prime}=2\left(K^{(h)}-\Lambda q^{2}-\frac{Q^{2}}{q^{2}}\right) \Phi, & q=q(\sigma), \\
\left(q^{2}\right)^{\prime \prime}=-2\left(K^{(h)}-\Lambda q^{2}-\frac{Q^{2}}{q^{2}}\right) \Phi, & q=q(\tau) .
\end{array}
$$

To integrate the derived equations, one has to express $\Phi$ through $q$ using equation (Dل moving the modulus sign.

We consider the static case $q=q(\sigma), \Phi>0$ and $q^{\prime}>0$ in detail. Then Eq. ([5]3) together with Eq. (Dلा) reduces to

$$
\left(q^{2}\right)^{\prime \prime}=2\left(K^{(h)}-\Lambda q^{2}-\frac{Q^{2}}{q^{2}}\right) q^{\prime}
$$

It can be easily integrated:

$$
\left(q^{2}\right)^{\prime}=2\left(K^{(h)} q-\frac{\Lambda q^{3}}{3}-2 M+\frac{Q^{2}}{q}\right)
$$

where $M=$ const is an integration constant, which coincides with mass in the Schwarzschild solution. Differentiating the left hand side and dividing it by $2 q>0$, we obtain equation

$$
q^{\prime}=K^{(h)}-\frac{2 M}{q}+\frac{Q^{2}}{q^{2}}-\frac{\Lambda q^{2}}{3} .
$$

Since $q^{\prime}=\Phi$ in the case under consideration, it implies expression for the conformal factor through variable $q$ :

$$
\Phi(q)=K^{(h)}-\frac{2 M}{q}+\frac{Q^{2}}{q^{2}}-\frac{\Lambda q^{2}}{3} .
$$

If $q=q(\sigma), \Phi>0$ and $q^{\prime}<0$, then the similar integration yields

$$
q^{\prime}=-\Phi(q)
$$

where the same conformal factor (5.15) stands in the right hand side. This case can be united with the previous one by re-writing equation for $q$ in the form

$$
\left|q^{\prime}\right|=\Phi(q), \quad q=q(\sigma), \quad \Phi>0 .
$$

The modulus sign in the left hand side means that if $q(\sigma)$ is a solution, then the function $q(-\sigma)$ is also the solution.

The static case for $\Phi<0$ is integrated in the same way:

$$
\left|q^{\prime}\right|=-\Phi(q), \quad q=q(\sigma), \quad \Phi<0 .
$$

If solution is homogeneous, $q=q(\tau)$ and $\Phi>0, q^{\prime}>0$, then integration of Eq. (5.14) yields the equality

$$
q^{\prime}=-\left(K^{(h)}-\frac{2 M}{q}+\frac{Q^{2}}{q^{2}}-\frac{\Lambda q^{2}}{3}\right)
$$


That is the conformal factor must be identified with the right hand side

$$
\hat{\Phi}=-\left(K^{(h)}-\frac{2 M}{q}+\frac{Q^{2}}{q^{2}}-\frac{\Lambda q^{2}}{3}\right) .
$$

We denote the expression for the conformal factor through $q$ by hat because in homogeneous case it differs by the sign. Thus, homogeneous solutions of Einstein's equations can be written in the form

$$
\begin{array}{lll}
\left|q^{\prime}\right|=\hat{\Phi}(q), & q=q(\tau), & \hat{\Phi}>0 . \\
\left|q^{\prime}\right|=-\hat{\Phi}(q), & q=q(\tau), & \hat{\Phi}<0 .
\end{array}
$$

If the conformal factor is negative, then the signature of the metric is $(-+--)$. In this case, we return to the previous signature (+- - ) after substitution $\tau \leftrightarrow \sigma$. This transformation allows us to unite static and homogeneous solutions by taking the modulus of the conformal factor in the expression for metric (5.6). Then a general solution of vacuum Einstein's equations with electromagnetic field ([.2) in the corresponding coordinate system takes the form

$$
d s^{2}=|\Phi|\left(d \tau^{2}-d \sigma^{2}\right)-q^{2} d \Omega^{2},
$$

where the conformal factor $\Phi$ is given by Eq. (5.55). Here the variable $q$ depends on $\sigma$ (static local solution) or $\tau$ (homogeneous local solution) through the differential equation

$$
\left|\frac{d q}{d \zeta}\right|= \pm \Phi(q)
$$

where the sign rule holds:

$$
\begin{aligned}
& \Phi>0: \quad \zeta=\sigma, \quad \text { the sign }+ \text { (static local solution), } \\
& \Phi<0 \text { : } \quad \zeta=\tau, \quad \text { the sign - (homogeneous local solution). }
\end{aligned}
$$

Thus the four-dimensional Einstein's equations imply that there is the metric with one Killing vector field on surface $\mathbb{U}$ which was considered in full detail in [ए6] . Now we can construct global solutions (maximally extended along geodesics) of vacuum Einstein's equations using the conformal block method. The number of singularities and zeroes of conformal factor (5.55) depends on relations between constants $K, M, Q$, and $\Lambda$. Therefore there are many qualitatively different global solutions.

Conformal factor (5.15) has one singularity: the second order pole at $q=0$. Therefore according to the rules formulated in [ए], [1]] every global solution correspond to one of the intervals $(-\infty, 0)$ or $(0, \infty)$. Because conformal factor $\Phi(q)$ is a smooth function for $q \neq 0$, all arising Lorentzian surfaces $\mathbb{U}$ and metrics on them are smooth. Totally, we have 33 topologically different solutions in the case B which are classified in [目].

\section{Changing topology of space in time}

Here we consider one new global spherically symmetric solution which describes changing topology of space sections during time evolution already at the classical level. It corresponds to the 
following values of constants:

$$
K^{(h)}=1, \quad \Lambda>0, \quad Q=\frac{1}{2 \sqrt{\Lambda}}, \quad M=\frac{1}{3} \sqrt{\frac{2}{\Lambda}} .
$$

Now the conformal factor (5.5) is

$$
\Phi(q)=-\frac{\sqrt{\Lambda}\left(q-\frac{1}{\sqrt{2 \Lambda}}\right)^{3}\left(\sqrt{\Lambda} q+\frac{3}{\sqrt{2}}\right)}{3 q^{2}} .
$$

It has triple zero at $q=1 / \sqrt{2 \Lambda}$. The corresponding four dimensional metric in Schwarzschild-like coordinates is

$$
d s^{2}=\Phi(q) d t^{2}-\frac{d q^{2}}{\Phi(q)}-q^{2}\left(d \theta^{2}+\sin ^{2} \theta d \varphi^{2}\right), \quad 0<q<1 / \sqrt{2 \Lambda} .
$$

The global solution is topologically the product $\mathbb{M}=\mathbb{U} \times \mathbb{S}^{2}$ where the Lorentzian surface $\mathbb{U}$ is depicted by the square Carter-Penrose diagram in Fig. $\mathrm{W}$. It has the de Sitter asymptotic at $q \rightarrow$ $\pm \infty$ where the surface is geodesically complete. The timelike boundaries $q=0$ are singular and geodesically incomplete. The very important fact is that the saddle point $q=1 / \sqrt{2 \Lambda}$ at the center denoted by the filled circle is geodesically complete. It is the intersection point of triple horizons.
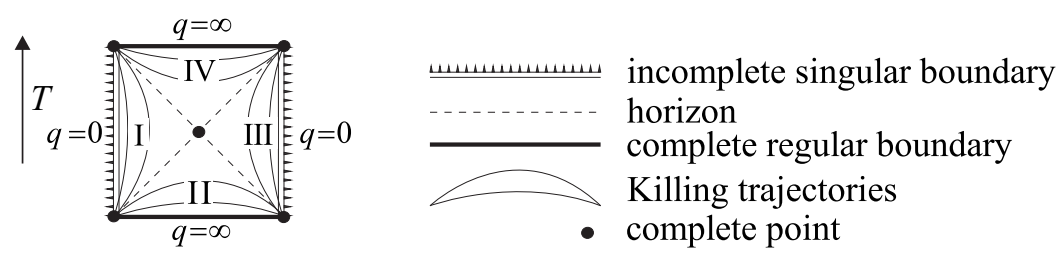

Figure 1: The Carter-Penrose diagram for triple horizon.

This Carter-Penrose diagram is interesting from physical standpoint. Namely, consider space sections of this diagram. If a section does not cross the saddle point in the center, then it is an interval of finite length with singular scalar curvature at the ends. If a section goes through the center then it is the union of two semi-infinite intervals because the complete point in the center is lying at space infinity. Now we introduce a global evolution parameter $T$, for example, vertical line on the diagram. Then the topology of space sections changes during evolution: for some value of $T$ we have two semi-infinite intervals instead of one interval of finite length. This example shows that changing topology of space in time may occur already at the classical level.

To visualize the surface, we show qualitatively the isometric embedding of surface $\mathbb{U}$ in threedimensional Minkowskian space-time $\mathbb{R}^{1,2}$ in Fig. $\downarrow$. Where the saddle point really lies at infinity.

\section{Conclusion}

We have found and classified all global solutions of general relativity with cosmological constant minimally coupled to an electromagnetic field assuming the space-time to be the warped product of two surfaces with Lorentzian and Euclidean signature metrics. The symmetry of solutions arises as the consequence of Einstein's equations ("spontaneous symmetry emergence"). 


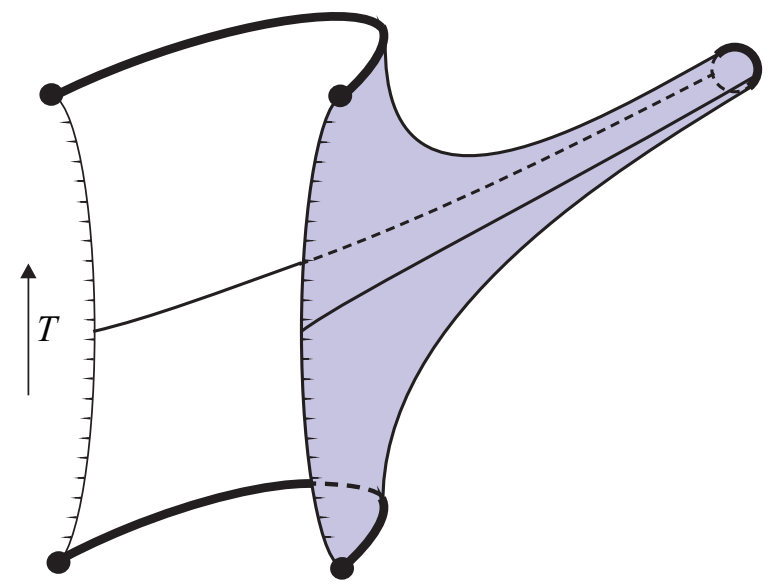

Figure 2: Qualitative isometric embedding of the Carter-Penrose diagram with triple horizon in threedimensional Minkowskian space-time.

Totally, there are 37 topologically inequivalent spatially symmetric solutions. New global solution describing changing topology of space sections in time is discussed in detail.

The work was supported in part by the Russian Government Program of Competitive Growth of Kazan Federal University (Russian Academic Excellence Project "5-100").

\section{References}

[1] D. Kramer, H. Stephani, M. MacCallum, and E. Herlt, Exact Solutions of the Einsteins Field Equations, Deutscher Verlag der Wissenschaften, Berlin 1980.

[2] M. D. Kruskal, Maximal extension of Schwarzschild metric, Phys. Rev., 119 (1960) 1743.

[3] G. Szekeres, On the singularities of a riemannian manifold, Publ. Mat. Debrecen, 7 (1960) 285.

[4] H. Reissner, Über die Eigengravitation des elektrischen Feldes nach der Einsteinschen Theorie, Ann. Physik (Leipzig), 50 (1916) 106.

[5] G. Nordström, On the energy of the gravitational field in Einstein's theory, Proc. Kon. Ned. Akad. Wet., 20 (1918) 1238.

[6] D. E. Afanasev and M. O. Katanaev, Global properties of warped solutions in general relativity with an electromagnetic field and a cosmological constant, Phys. Rev., D100 (2019) 024052 [physics.gen-ph/1904.04648].

[7] M. Walker, Block diagrams and the extension of timelike two-surfaces, J. Math. Phys., 11 (1970) 2280.

[8] B. Carter, Black hole equilibrium states, in C. DeWitt and B. C. DeWitt, editors, Black Holes, Gordon \& Breach, New York, pages 58-214 (1973).

[9] M. O. Katanaev, All universal coverings of two-dimensional gravity with torsion, J. Math. Phys., 34 (1993) 700.

[10] T. Klösch and T. Strobl, Classical and quantum gravity in $1+1$ dimensions: II. The universal coverings, Class. Quantum Grav., 13 (1996) 2395. 
[11] T. Klösch and T. Strobl, Classical and quantum gravity in $1+1$ dimensions: III. Solutions of arbitrary topology, Class. Quantum Grav., 14 (1997) 1689.

[12] C.-G. Huang and C.-B. Liang, A torus like black hole, Phys. Lett., A201 (1995) 27.

[13] J. P. S. Lemos, Cylindrical black hole in general relativity, Phys. Lett., B353 (1995) 46.

[14] D. R. Brill, J. Louko, and P. Peldan. Thermodynamics of (3+1)-dimensional black holes with toroidal or higher genus horizons, Phys. Rev., D56 (1997) 3600.

[15] M. O. Katanaev, T. Klösch, and W. Kummer. Global properties of warped solutions in general relativity, Ann. Phys., 276 (1999) 191.

[16] M. O. Katanaev, Global solutions in gravity. Lorentzian signature, Proc. Steklov Inst. Math., 228 (2000) 158.

[17] M. O. Katanaev, Geometric methods in mathematical physics, ver. 3, (2016) [math-ph/1311.0733] [in Russian].

[18] G. D. Birkhoff, Relativity and modern physics, Harvard University Press, Cambridge 1923.

[19] J. T. Jebsen, Uber die allgemeinen kugelsymmetrischen losungen der einsteinschen gravitationsgleichungen im vakuum, Ark. Mat. Ast. Fys., 15 (1921) 1.

[20] H. Weyl, Zur gravitationstheorie, Ann. Physik, 54 (1917) 117.

[21] G. A. Korn and T. M. Korn, Mathematical Handbook, McGraw-Hill Book Company, New York London 1968.

[22] M. O. Katanaev, Effective action for scalar fields in two-dimensional gravity, Ann. Phys., 296 (2002) 1. 